\title{
Surface-Normal Plasmonic Modulator using Sub-Wavelength Metal Grating on Electro-Optic Polymer Thin Film
}

\author{
Fanghui Ren ${ }^{\mathrm{a}}$, Ming Li ${ }^{\mathrm{b}}$, Qian Gao ${ }^{\mathrm{a}}$, William Cowell, III', Jingdong Luo ${ }^{\mathrm{b}}$, Alex K-Y Jen ${ }^{\mathrm{b}}$, Alan X.
} Wang ${ }^{\text {a, * }}$

${ }^{\mathrm{a}}$ School of Electrical Engineering and Computer Science, Oregon State University, Corvallis, OR, 97331, USA

${ }^{\mathrm{b}}$ Department of Materials Science \& Engineering, University of Washington, Seattle, WA 98195, USA

${ }^{\mathrm{c}}$ Department of Chemistry, Oregon State University, Corvallis, OR, 97331, USA

${ }^{*}$ Corresponding Author e-mail address: wang@eecs.oregonstate.edu

\begin{abstract}
In this work, we present the design, fabrication, and characterization of a surface-normal plasmonic modulator using sub-wavelength metal grating. The device consists of a $1.77 \mu \mathrm{m}$ efficiently

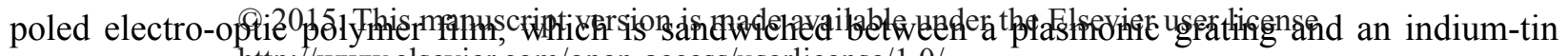
http://www.elsevier.com/open-access/userlicense/1.0/

oxide film. A strong Fano resonance with a sharp plasmonic bandgap at $1522 \mathrm{~nm}$ wavelength was observed and electro-optical modulation was demonstrated based on the refractive index modulation of the polymer film. With future improvement in lowering the driving voltage and increasing the modulation depth, this hybrid organic/plasmonic modulator can readily expand into modulator arrays for three dimensional optical interconnects.
\end{abstract}

Key Words: Electro-optical modulator; polymer; surface plasmons; Fano resonance 


\section{Introduction}

High speed Electro-optic (E-O) modulators have been identified as the key components in optical transceivers for communication systems [1-3]. Plasmonic modulators based on surface-plasmon polaritons (SPPs) will play pivotal roles in enabling nano-scale on-chip photonics for high-density integration [4-10]. SPP based periodic metallic structures have been intensively investigated as active plasmonic devices in modulation applications, as they provide strong optical mode confinement, ultracompact footprint, and active manipulation of photons [11-14]. In recent years, dynamic control over SPPs has been realized by modulating the refractive index of the dielectric layer adjacent to the metal surface [15-16]. Among the choices of the materials, E-O polymer possesses exclusive advantages such as large Pockels effect $(>200 \mathrm{pm} / \mathrm{V})$, fast modulation speed and low dispersion across the RF to optical frequencies [17-21]. Further, polymers can be infiltrated into plasmonic structures, which allow the combination of the highly nonlinear optical effect of polymers with the strong field confinement of ultra-

compact plasmonic structures. Recently, plasmonic/polymer hybrid waveguide modulators have been demonstrated, which are usually formed by uniform metal-dielectric thin films or stripes [22-25]. However, such modulators only allow in-plane photon manipulation with high insertion loss.

In this work, we present the design, fabrication, and characterization of a surface-normal plasmonic/EO polymer modulator that combines the merits of sub-wavelength metal gratings and efficiently poled EO polymer thin film. The reported modulator structure possesses high optical confinement, low insertion loss, and is easy integrated with other optoelectronic devices for free-space 3-D optical interconnects $[26,27]$.

\section{Device Structure}

The configuration of the device is shown in Figure 1(a), which consists of a gold (Au)/polymer / indium-tin oxide (ITO) sandwich structure on a glass substrate. A layer of $110 \mathrm{~nm}$ ITO film was deposited onto a $25 \mathrm{~mm} \times 25 \mathrm{~mm}$ glass substrate using a two-inch ITO target $\left(90 \% \mathrm{In}_{2} \mathrm{O}_{3}, 10 \% \mathrm{SnO}_{2}\right.$ by weight) in an AJA Orion 5 sputtering system. Substrate rotation was employed to ensure uniform film thickness. Deposition was performed at $75 \mathrm{~W}$ of RF power and a chamber pressure of 5 mTorr using 9.7 
sccm of argon and $0.3 \mathrm{sccm}$ of oxygen. Substrates were heated to $300{ }^{\circ} \mathrm{C}$ during the deposition. The ITO film thickness was measured on photolithographically patterned features using a Tencor Alpha Step 500 profilometer. The resistivity was measured to be $334 \mu \Omega \bullet \mathrm{cm}$ using a Jandel Multi Height Probe with a RM2 Resistivity Test Unit. The ITO electrode was patterned and partially etched by hydrochloric acid in order to reduce the capacitance with the Au grating. The E-O polymer in this study is a guest-host polymer composite in poly (methyl methacrylate-co-styrene) (PMMA-co-PS) using AJLZ53 as one of most efficient and photo-stable dipolar tetraene chromophores at the loading level of $15 \mathrm{wt} \%$. This polymer exhibits large E-O coefficient and high processing reliability in poled thin films, and has been used as a standard EO polymer with glass transition temperature $\left(T_{g}\right)$ of $110{ }^{\circ} \mathrm{C}$ for the study of photonic devices [28]. Its thin film was prepared by spin-coating of formulated solution in dibromomethane onto the ITO substrate with a spin speed at $2000 \mathrm{rpm}$ for 60 seconds, followed by overnight baking in vacuum oven at $65{ }^{\circ} \mathrm{C}$ to ensure complete removal of the residual solvent, leading to high optical quality films with thickness of $1.77 \mu \mathrm{m}$. The refractive index of the unpoled polymer films was measured by ellipsometry to be 1.571 at $1522 \mathrm{~nm}$. A $100 \mathrm{~nm}$ Au thin film was deposited onto the polymer layer by thermal evaporation with a deposition rate of $8 \AA$ /sec. In order to reduce the RC delay of the device, a shadow mask was used to reduce the gold pad size. The key for enabling modulation is to create the Pockels effect in the E-O polymer sandwiched between the top Au and bottom ITO layers. This was achieved by generating the noncentrosymmetric order of nonlinear optical chromophores through poling process in presence of applied electric field. The E-O polymer was poled with contact poling method with E-O coefficient $r_{33}$ of $71 \mathrm{pm} / \mathrm{V}$ as measured by the standard Teng-man reflective measurement system at $1300 \mathrm{~nm}$ [29]. The nano-scale slits were milled by focused-ion beams (FIB), with gallium ion energy of $30 \mathrm{kV}$ and current of $10 \mathrm{pA}$. The periodicity of the grating was designed at $978 \mathrm{~nm}$ with the slit width of $100 \mathrm{~nm}$, as shown in Figure 1(b).

The key challenge faced in the fabrication process is precisely controlling geometrical dimensions and obtaining smooth slits at the Au/polymer interface. The ability to control the width of the gap and to minimize the fabrication defects is crucial for obtaining a sharp bandgap for the device. An optical image of the fabricated $53.8 \mu \mathrm{m} \times 53.8 \mu \mathrm{m}$ plasmonic grating structure after the FIB milling is shown in Figure 2(a). A high-resolution scanning electron microscopy (SEM) image of the grating is shown in Figure 2(b), 
which indicates the high quality of the grating structure with smooth slits in the presence of E-O polymer thin film.

\section{Experiment and Results}

\subsection{Simulation and Experiment of Fano resonance}

The Fano resonances in the sub-wavelength metal grating have been intensively investigated by many researchers [11-14]. Figure 3(a) shows the experimental setup of measuring the transmission spectrum. A broadband continuous wave laser (Thorlabs, S1FC1550) centered at $1550 \mathrm{~nm}$ was coupled into a fiberbased polarizer (Thorlabs, ILP1550SM-FC) to generate a linearly transverse magnetic (TM) polarized light with electric field polarized perpendicular to the slit direction (along $\mathrm{x}$ axis in Figure 2). The output light was then collimated through a $40 \times$ objective lens $(\mathrm{NA}=0.65)$. The diameter of the collimated beam is about $130 \mu \mathrm{m}$. A $50 \mu \mathrm{m}$ pinhole was used in front of the modulator to block the excessive light of the collimated beam before coupling to the device in order to obtain the coupling efficiency to the SPP mode.

The device was mounted on a five axis stage, which allowed precise adjustment of the position as well as the incident angle within $5 \times 10^{-5} \mathrm{rad}$. The transmitted beam after the sample was focused by a $40 \times$ objective lens and coupled into an optical spectrum analyzer (Thorlabs, OSA203B) with a standard SMF28 fiber.

Figure 3(b) shows the measured and simulated transmission spectra with a wavelength range from $1450 \mathrm{~nm}$ to $1600 \mathrm{~nm}$. The simulation is based on Rigorous Coupled Wave Analysis (RCWA) in DiffractMod of Rsoft ${ }^{\mathrm{TM}}$. The transmission spectra, indicate the asymmetric lineshape of the Fano resonance with a sharp transition edge. The resonance is attributed to the coupling of the discrete guided modes induced by Bragg-grating-modulated SPPs with the Fabry-Perot (FP) resonance in the narrow slits and waveguide mode resonance in the polymer layer $[11,12]$. The location of the transmission maximum agrees with the simulated curve, as shown in Figure 3(b). Our experimental results show that the total transmitted optical power is $23 \%$, which is $6.3 \mathrm{~dB}$ insertion loss. In our simulation in Fig. 3(b), the structure shows $43 \%$ of normalized transmission at the resonance peak, where the loss is determined by the combined loss from the metallic grating and the absorption from the ITO film. The optical loss of the 
$100 \mathrm{~nm}$ ITO thin film was measured to be $1.1 \mathrm{~dB}$ at $1528 \mathrm{~nm}$. From the experimental results, it can be concluded that the coupling of the light to the SPP mode of the plasmonic grating shows a loss of $5.2 \mathrm{~dB}$. The reduced peak intensity and broadening of the spectrum compared with simulation results are attributed to the non-perfect collimation, the edge roughness from the FIB process, and the ohmic loss of the $\mathrm{Au}$ thin film. The resistivity the $100 \mathrm{~nm} \mathrm{Au}$ thin film was measured to be $4.08 \times 10^{-8} \Omega * \mathrm{~m}$, which is nearly two times higher than bulk Au resistivity [30]. Figure 3(c) and Figure 3(d) show the cross-

sectional views of the electric field distribution $\left|E / E_{0}\right|$ (where $E_{0}$ is the electric field amplitude of the incident light) associated with the SPPs at the Au-polymer interface at $1522 \mathrm{~nm}$ along the z-axis and xaxis, respectively with no applied voltage. The enhanced $E_{z}$ extend into the polymer layer, as shown in Figure 3(c), resulting in overall $3 \times$ electric field amplitude enhancements over a large volume in the polymer layer, which has very good overlap with the modulating electric field induced by the vertical electrodes. Figure 3(d) indicates extraordinary transmission effects in the narrow slits.

\subsection{Demonstration of electro-optical modulation}

In order to investigate the E-O modulation due to the Pockels effect of the polymer layer, a 20V DC voltage was applied between the grating and the grounded ITO layer. The change of the refractive index $\Delta n$ can be written as [31]

$$
\Delta n=-\frac{1}{2} n_{\mathrm{e}}{ }^{3} r_{33} \frac{V}{d}
$$

where $V$ is the applied voltage and $d$ is the polymer film thickness. The extraordinary refractive index $n_{e}$ of the poled films is 1.61 measured by Metricon 2010 prism coupler. Considering the results that $r_{33}$ is 71 $\mathrm{pm} / \mathrm{V}$ at $1300 \mathrm{~nm}$ corresponds to $60 \mathrm{pm} / \mathrm{V}$ at $1522 \mathrm{~nm}$ based on the two-level approximation [32], $\Delta n$ was calculated as 0.0014 . The wavelength of the Fano resonance is determined by the periodicity of the structure $p$, Au permittivity $\varepsilon_{A u}$, and the polymer permittivity $\varepsilon_{d}$, which can be explained by the SPP zeroorder relation [33]:

$$
\lambda_{0}=n_{s p p} p
$$




$$
\text { where } n_{s p p}=\operatorname{Re}\left(\sqrt{\frac{\varepsilon_{A u} \varepsilon_{d}}{\varepsilon_{A u}+\varepsilon_{d}}}\right)
$$

The simulation curves in Figure 4 indicate a wavelength shift occurs due to the small change of polymer index $\Delta n$ of 0.0017 in the presence of the applied voltage. With a probing wavelength of 1522 $\mathrm{nm}$, the simulated transmission spectra show that a very small index modulation of 0.0017 induces the decrease of the optical transmission from $17.5 \%$ to $12.6 \%$. Figure 4 also shows experimental results of the wavelength shift with an external $20 \mathrm{~V}$ voltage applied. The measurement curves show that the optical transmission drops from $15.3 \%$ to $14.1 \%$ at $1522 \mathrm{~nm}$. The discrepancy between the experiment and simulation possibly result from the degradation of the polymer E-O efficiency $r_{33}$ in the grating region after the FIB process. Due to the small thermal conductivity of polymers[34], FIB heating could induce thermal relaxation of poled films to certain extend, and therefore the $r_{33}$ value of the grating region (53.8 $\mu \mathrm{m} \times 53.8 \mu \mathrm{m})$ is degraded compared to the measured value using the Teng-man method with a large collimated beam size $(2.6 \mathrm{~mm} \times 2.6 \mathrm{~mm})$. Thin film E-O measurement proves that poled films, after being exposed to thermal excursion at $60{ }^{\circ} \mathrm{C}$ for 15 minutes, retains about $70 \%$ of its initial $r_{33}$ values. From the experimental result of wavelength shift in Figure 4, the change of the refractive index was estimated to be 0.00063 , which results in an in-device $r_{33}$ of $27 \mathrm{pm} / \mathrm{V}$ at $1522 \mathrm{~nm}$ based on Equation (1). The change of the optical field distribution at the probing wavelength induced by the modulation of the dielectric layer refractive index can be found in Ref 11-14 and our previous work in Ref 16.

A tunable laser (HP 8168A) at $1522 \mathrm{~nm}$ was used in order to characterize the E-O modulation. An InGaAs photodetector was connected to an oscilloscope in order to monitor the transmitted optical power. The photocurrent is directly converted to voltage output through the $50 \Omega$ impedance of an oscilloscope without any amplifier. A $1 \mathrm{kHz}$ sinusoidal wave (20V peak-to-peak) was applied to the device from a function generator (Wavetek, Model 395). The output of the optical signal in response to the electric driving signal is shown in Figure 5. The modulation depth, which is the amplitude of the modulation voltage divided by the reference signal, only achieves $7.2 \%$ in our experiment, however, it is highly 
possible to obtain large E-O modulation $(>50 \%)$ by using more efficient $\left(r_{33}>200 \mathrm{pm} / \mathrm{V}\right)$ E-O polymers with higher thermal stabilities. The frequency response of our structure is limited majorly by the capacitance of the device, which is $260 \mathrm{pF}$ measured at $100 \mathrm{k} \mathrm{Hz}$. The relatively large capacitance is attributed to large gold pad size $(3 \mathrm{~mm} \times 3 \mathrm{~mm})$. Future work will also be focused on optimizing the FIB fabrication condition to obtain sharper transition edge of the Fano Resonance, which will increase the modulation depth. Modulation with lower driving voltage can be achieved by decreasing the thickness of the E-O polymer layer [35]. By these optimizations in design and fabrication, it would be possible to reduce the driving voltage to $\sim 1 \mathrm{~V}$ and achieve a modulation depth of $\sim 3 \mathrm{~dB}$.

\section{Conclusion}

In conclusion, we have designed and fabricated an electrically driven surface-normal plasmonic/E-O polymer modulator that is suitable for 3-D optical interconnects. Due to the extraordinary transmission, the hybrid plasmonic/polymer device exhibits a Fano resonance with a sharp transition edge. The E-O effect in the polymer film enables modulation of the Fano resonance. Further improvements of the modulation performance will be focused on optimization of the poling process, decreasing the polymer thickness with more efficient and thermally stable E-O polymers, and improving the FIB fabrication, which will lead to an ultra-compact device with fast modulation and low driving voltage. Such surfacenormal plasmonic modulators are expandable to 2-D arrays, and can be integrated with energy-efficient optoelectronic driving circuits and low-cost continuous-wave vertical surface-emitting lasers towards the realization of out-of-plane optical interconnects.

\section{Acknowledgement}

A. X. Wang acknowledges support by the National Science Foundation under grant No. 1342318. A. K-Y Jen thanks the Air Force Office of Scientific Research (AFOSR FA9550-09-1-0426, and FA8650-12-M5130 under the Small Business Technology Transfer Research (STTR) program) and the Boeing-Johnson 
Foundation for the support. The authors would like to thank Dr. Sean Muir for the deposition of the ITO thin film.

\section{References}

[1] G. T. Reed, G. Mashanovich, , F. Y. Gardes, and D. J. Thomson, Silicon optical modulators, Nature Photon. 4 (2010) 518-526.

[2] B. Bortnik, Y.-C. Hung, H. Tazawa, B.-J. Seo, J. Luo, A. K.-Y. Jen, W. H. Steier, and H. R. Fetterman, Electrooptic Polymer Ring Resonator Modulation up to $165 \mathrm{GHz}$, IEEE J. Sel. Topics Quantum Electron. 13 (2007) $104-110$.

[3] L. Liao, A. Liu, D. Rubin, J. A. B. J. Basak, Y. A. C. Y. Chetrit, H. A. N. H. Nguyen, R. A. C. R. Cohen, N. A. I. N. Izhaky, and M. A. P. M. Paniccia, 40 Gbit/s silicon optical modulator for high-speed applications, Electron. Lett. 43 (2007) 1196-1197.

[4] D. K. Gramotnev, and S. I. Bozhevolnyi, Plasmonics beyond the diffraction limit, Nature Photon. 4 (2010) 83-91.

[5]R. F. Oulton, V. J. Sorger, D. A. Genov, D. F. P. Pile, and X. Zhang, BA hybrid plasmonic waveguide for subwavelength confinement and long-range propagation, Nat. Photon. 2 (2008) 496-500.

[6]W. Cai, J. S. White, and M. L. Brongersma, Compact, high-speed and power-efficient electrooptic plasmonic modulators, Nano Lett. 9 (2009) 4403-4411 .

[7]E. Ozbay, Plasmonics: merging photonics and electronics at nanoscale dimensions, Science 311 (2006) 189-193.

[8]X. M. Sun, L. J. Zhou, X. W. Li, Z. H. Hong, and J. P. Chen, Design and analysis of a phase modulator based on a metal-polymer-silicon hybrid plasmonic waveguide, Appl. Opt. 50 (2011) 34283434.

[9]T. Nikolajsen, K. Leosson, S. I. Bozhevolnyi, Surface plasmon polariton based modulators and switches operating at telecom wavelengths, Appl. Phys. Lett. 85 (2004) 5833-5835. 
[10]D. Pacifici, H. J. Lezec, and H. A. Atwater, All-optical modulation by plasmonic excitation of CdSe quantum dots, Nature Photon. 1 (2007) 402-406.

[11]A. Christ, T. Zentgraf, and J. Kuhl, Optical properties of planar metallic photonic crystal structures: Experiment and theory, Phys. Rev. B 70 (2004) 125113.

[12]J. A. Porto, F. J. García-Vidal, and J. B. Pendry, Transmission resonances on metallic gratings with very narrow slits, Phys. Rev. Lett. 83 (1999) 2845.

[13]G. D’Aguanno, N. Mattiucci, M. J. Bloemer, D. De Ceglia, M. A. Vincenti, and A. Alù, Transmission resonances in plasmonic metallic gratings, J. Opt. Soc. Am. B 28 (2011) 253-264 .

[14]B. Tang, L. Dai, and C. Jiang, Transmission enhancement of slow light by a subwavelength plasmon-dielectric system, J. Opt. Soc. Am. B 27 (2010) 2433-2437.

[15]T. Nikolajsen, K. Leosson, S. I. Bozhevolnyi, Surface plasmon polariton based modulators and switches operating at telecom wavelengths, Appl. Phys. Lett. 65 (2004) 5833-5836.

[16]F. Ren, X. Wang, and A. X. Wang, Thermo-optic modulation of plasmonic bandgap on metallic photonic crystal slab, Appl. Phys. Lett. 102 (2013)181101.

[17]Y. Enami, C. T. Derose, D. Mathine, C. Loychik, C. Greenlee, R. A. Norwood, T. D. Kim, J. Luo, Y. Tian, A. K.-Y. Jen, and N. Peyghambarian, Hybrid polymer/sol-gel waveguide modulators with exceptionally large electro-optic coefficients, Nature Photon. 1(2007) 180-185.

[18]H. Ma, A. K-Y. Jen, and L. R. Dalton, Polymer-based optical waveguides: materials, process, and devices, Adv. Mater. 14 (2002) 1339-1365.

[19]C. Y. Lin, X. Wang, S. Chakravarty, B. S. Lee, W. Lai, J. Luo, A. K-Y. Jen, and R. T. Chen, Electro-optic polymer infiltrated silicon photonic crystal slot waveguide modulator with $23 \mathrm{~dB}$ slow light enhancement, Appl. Phys. Lett. 97 (2010) 093304.

[20]F. E. Doany, C. L. Schow, C. W. Baks, D.M. Kuchta, P. Pepeljugoski, L. Schares, R. Budd, F. Libsch, R. Dangel, F. Horst, B. J. Offrein, and J. A. Kash, 160 Gb/s bidirectional polymer-waveguide board-level optical interconnects using CMOS-based transceivers, IEEE Trans. Advanced Packaging $32(2009) 345-359$. 
[21]R. Himmelhuber, O. D. Herrera, R. Voorakaranam, L. Li, A. M. Jones, R. A. Norwood, J. Luo, A. K.-Y. Jen, and N. Peyghambarian, A silicon-polymer hybrid modulator-Design, simulation and proof of principle, J. Lightwave Technol. 31 (2013) 4067-4072.

[22]D. Dai, and S. He, A silicon-based hybrid plasmonic waveguide with a metal cap for a nano-scale light confinement, Opt. Exp. 17 (2009) 16646-16653.

[23]S. Randhawa, S. Lachèze, J. Renger, A. Bouhelier, R. E. de Lamaestre, A. Dereux, and R. Quidant. Performance of electro-optical plasmonic ring resonators at telecom wavelengths, Opt. Exp. 20 (2012) $2354-2362$.

[24]A. Melikyan, L. Alloatti, A. Muslija, D. Hillerkuss, P. C. Schindler, J. Li, R. Palmer, . Korn, S. Muehlbrandt, D. Van Thourhout, B. Chen, R. Dinu, M. Sommer, C. Koos, M. Kohl, W. Freude and J. Leuthold, High-speed plasmonic phase modulators, Nature Photon. 8 (2014) 229-233.

[25]J. Schildkraut, Long-range surface plasmon electrooptic modulator, Appl. Opt. 27 (1988) 45874590.

[26]E. Mohanmmed, A. Alduino, T. Thomas, H. Braunisch, D. Lu, J. Heck, A. Liu, I. Young, B. Barnett, G. Vandentop, R. Mooney, Optical interconnect system integration for ultra-short-reach applications, J. Intel. Technol. 8 (2004) 115-127.

[27]A. Olivieri, C. Chen, S. Hassan, E. Lisicka-Skrzek, N. Tait, and P. Berini. "Plasmonic nanostructured metal-oxide-semiconductor reflection modulators." Nano lett. 15(2015) 2304-2311 .

[28]S. Huang, J. Luo, H.-L. Yip, A. Ayazi , X.-H. Zhou, M. Gould, A. Chen, T. Baehr-Jones, M. Hochberg, and A. K.-Y. Jen, Efficient poling of electro-optic polymers in thin films and silicon slot waveguides by detachable pyroelectric crystals, Adv. Mater. 24(2012) OP42.

[29]C. C. Teng and H. T. Man, Simple reflection technique for measuring the electro - optic coefficient of poled polymers, Appl. Phys. Lett. 56(1990) 1734.

[30] R. A. Matula, Electrical resistivity of copper, gold, palladium, and silver. J. Phys. Chem. Ref. Data. 8 (1979)1147-1298. 
[31]R. W. Boyd, Nonlinear optics, second ed., Academic press, 2008.

[32]K. D. Singer, M. G. Kuzyk, \& J. E. Sohn, Second-order nonlinear-optical processes in orientationally ordered materials: relationship between molecular and macroscopic properties, JOSA B 4 (1987) 968-976.

[33]H.Raether, Surface polaritions on smoooth and rough surfaces and on gratings, Springer-Verlag, Berlin, 1988.

[34]S. Kim, M. J. Park, N. P. Balsara, G. Liu, and A. M. Minor, Minimization of focused ion beam damage in nanostructured polymer thin films, Ultramicroscopy 111 (2011) 191-199.

[35]Y. Jouane, Y. C. Chang, D. Zhang, J. Luo, A. Jen, and Y. Enami, Unprecedented highest electrooptic coefficient of $226 \mathrm{pm} / \mathrm{V}$ for electro-optic polymer/TiO2 multilayer slot waveguide modulators, Opt. Exp. 22 (2014) 27725-27732. 
(a)

(b)

Glass substrate

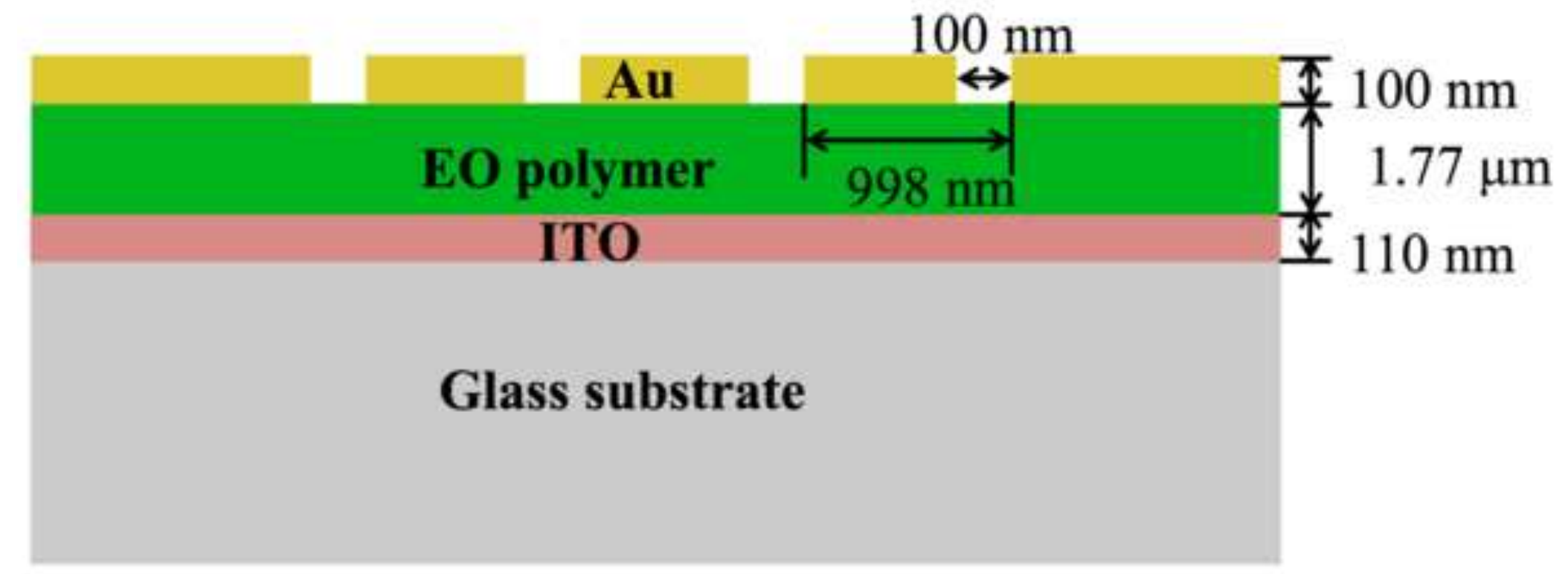

\section{ITO electrode}

\title{
EO polymer
}

(n)

Au grating

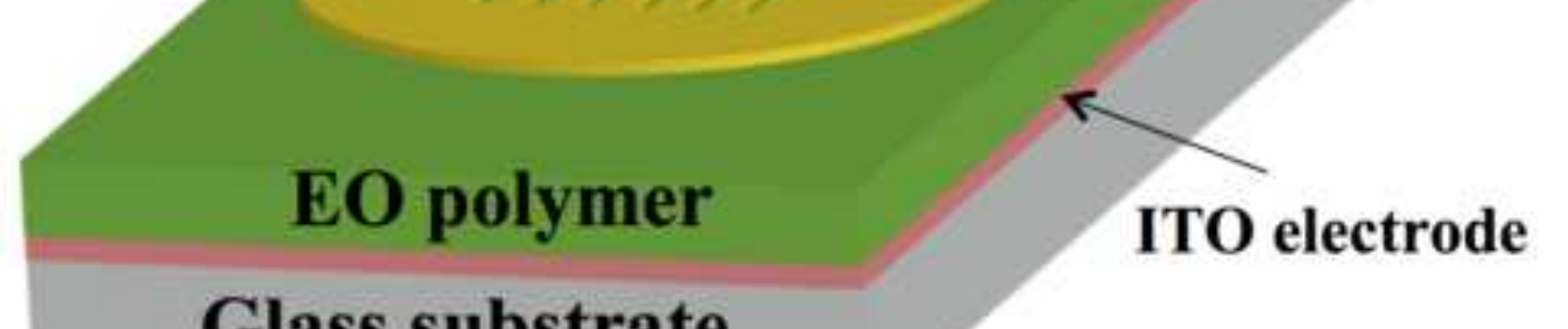

\author{
Glass substrate
}


$\stackrel{y}{i}$

(a)

$10 \mu \mathrm{m}$

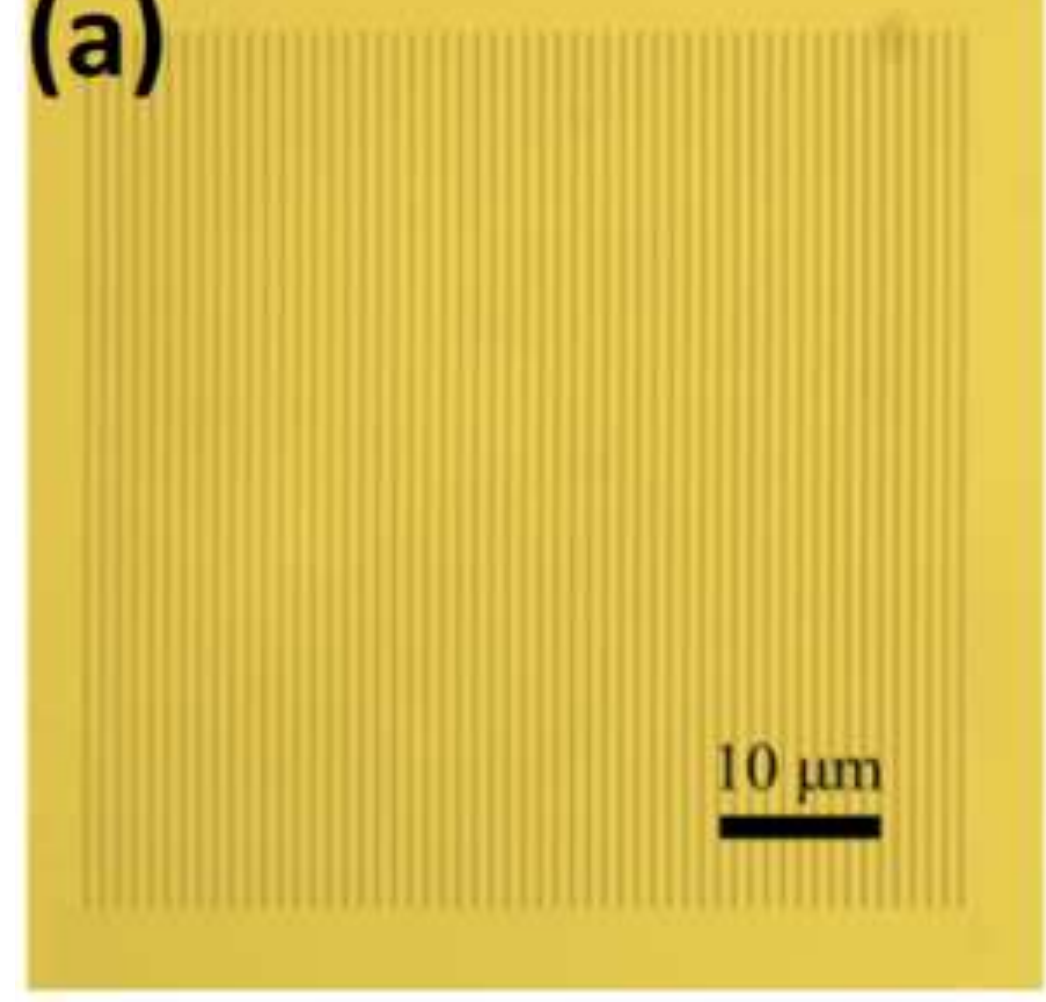

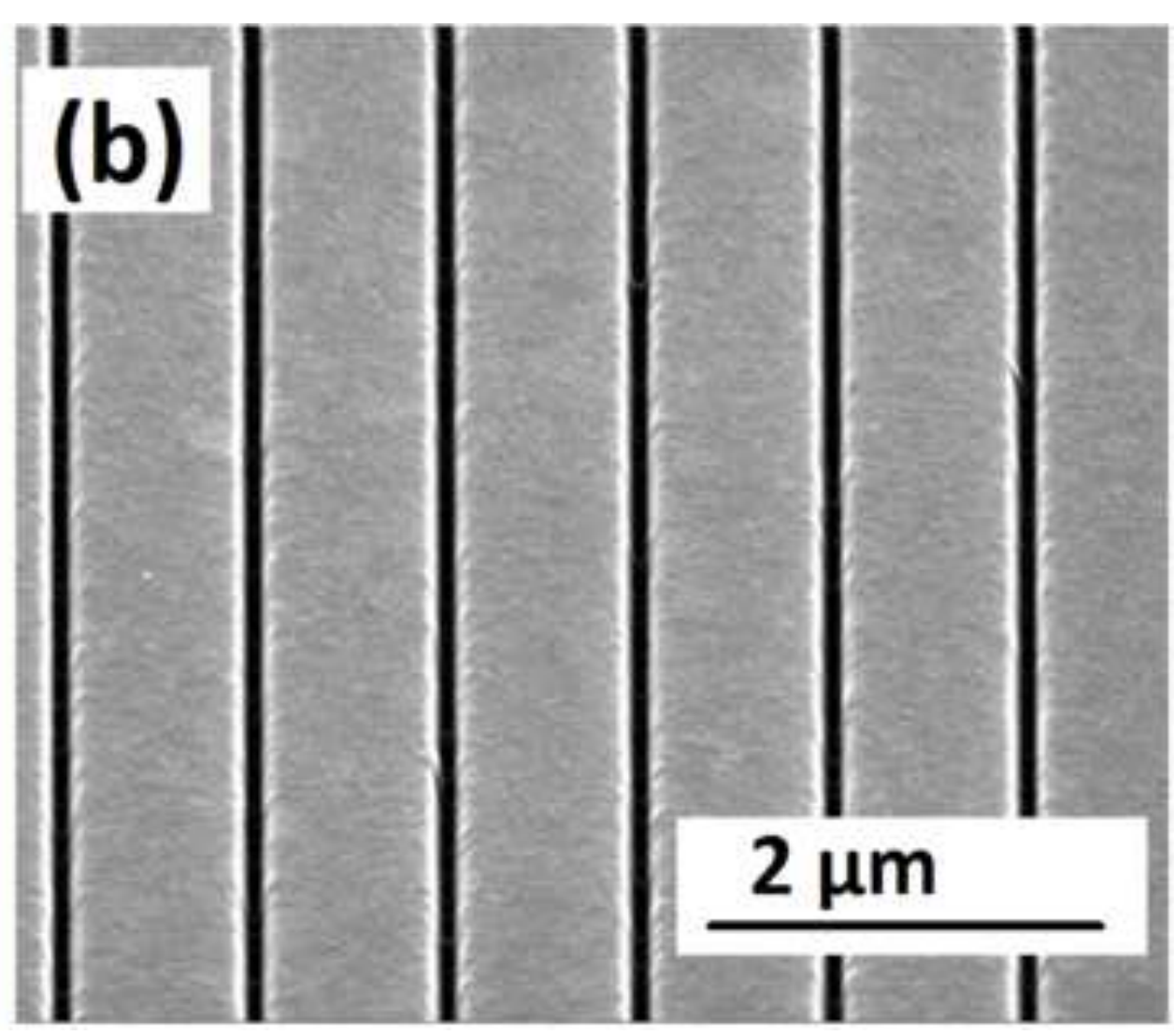

(b)

$2 \mu \mathrm{m}$

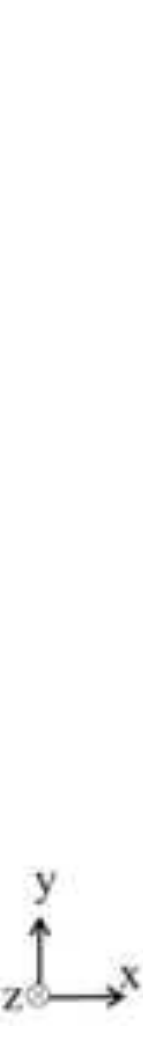




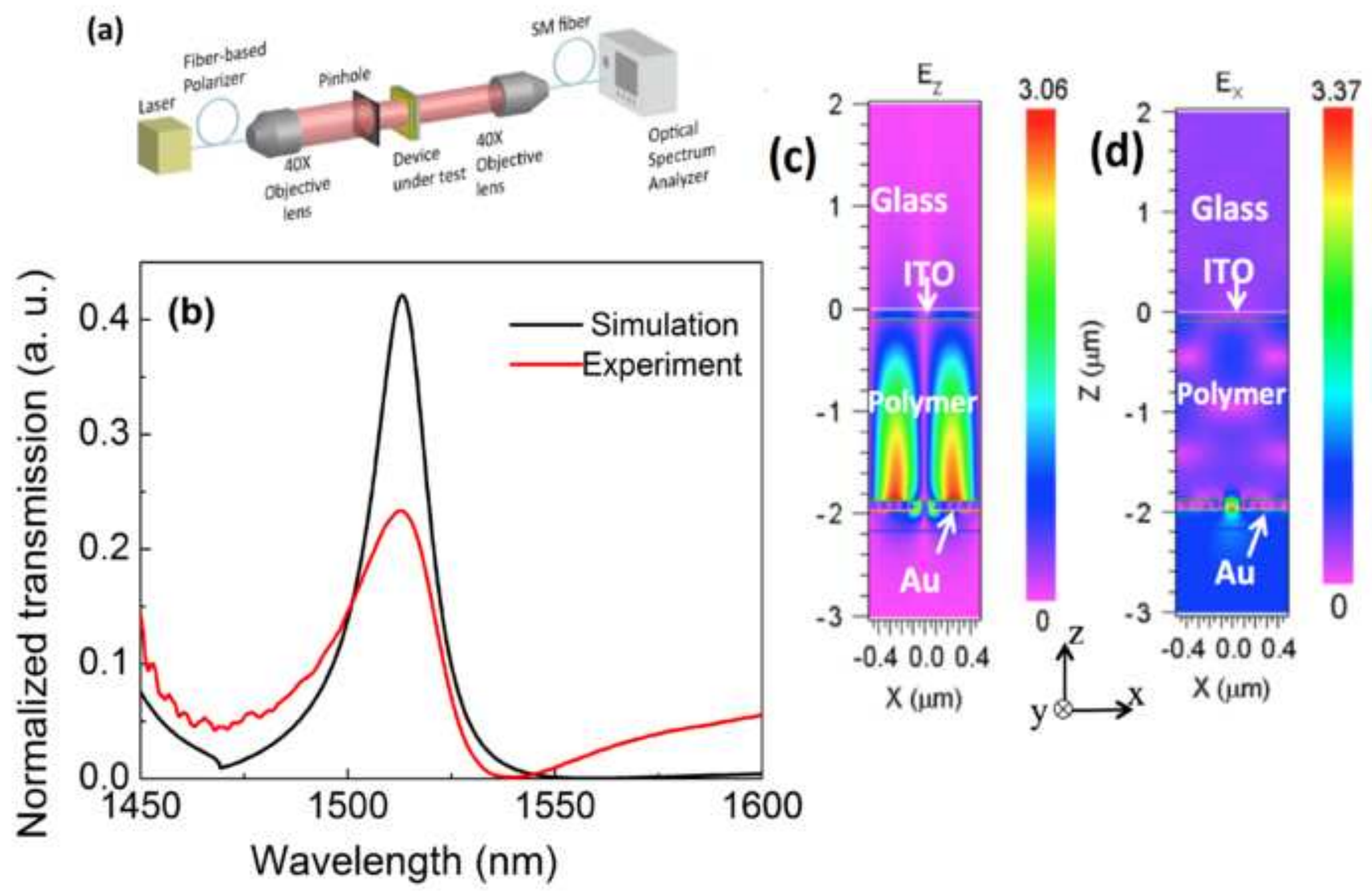




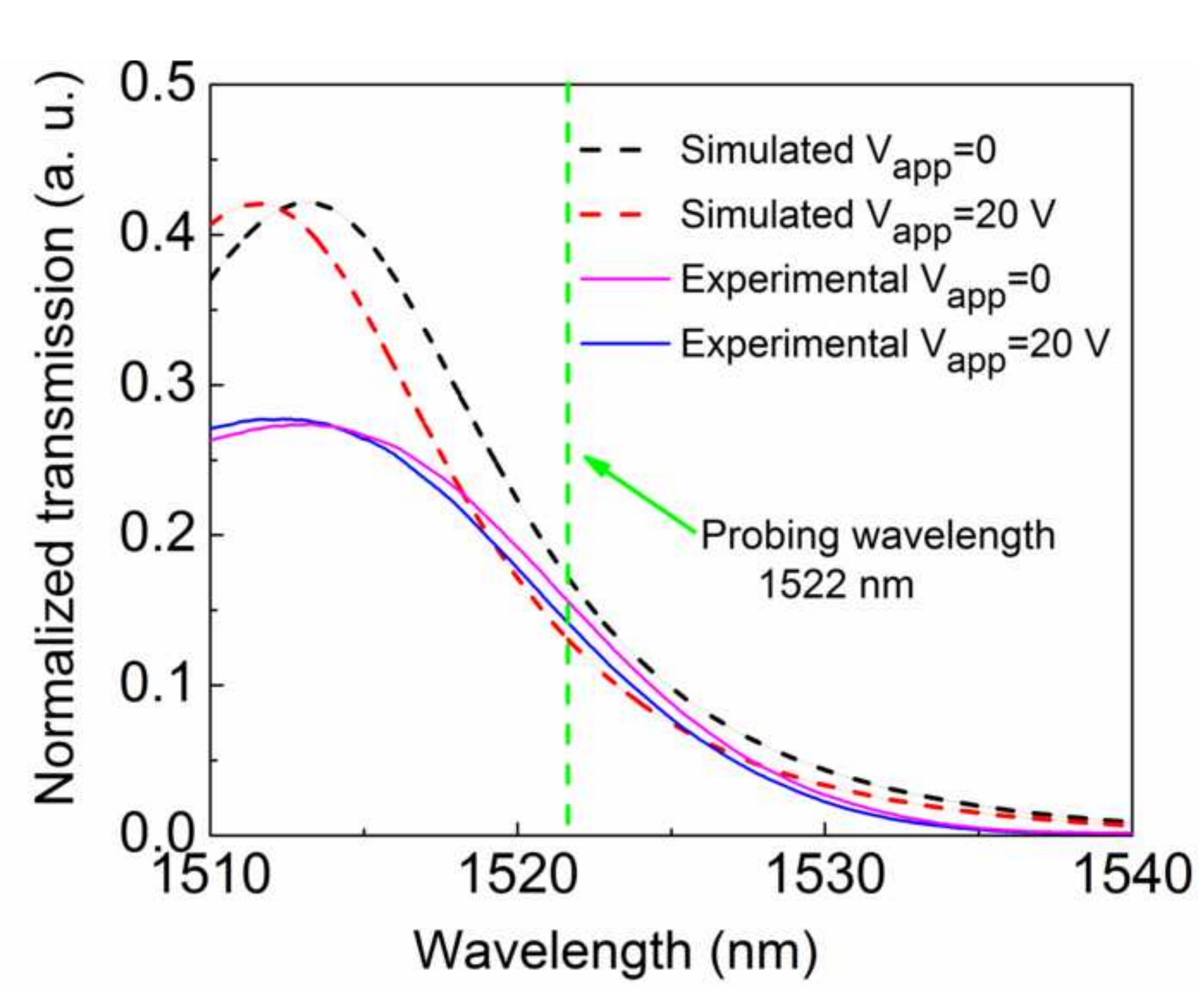




\section{Figure Captions:}

Fig.1.(a) Schematic illustration of the surface-normal plasmonic modulator (b) Cross-sectional view of the device structure with geometric parameters

Fig.2. (a) Optical microscopy and (b) SEM images showing the fabricated sub-wavelength metal grating

Fig.3. (a) Experimental setup for optical transmission spectrum. (b) The simulation and experimental curves showing the optical transmission spectrum. The Electric field distribution along (c) $\mathrm{z}$ direction and (d) $\mathrm{x}$ direction at $1522 \mathrm{~nm}$.

Fig.4. The simulation and experimental curves showing the spectrum shift under 20V voltage

Fig.5. Electro-optical modulation of the Fano resonance: lower curve is the driving electric signal, and upper curve is the responding optical signal. 